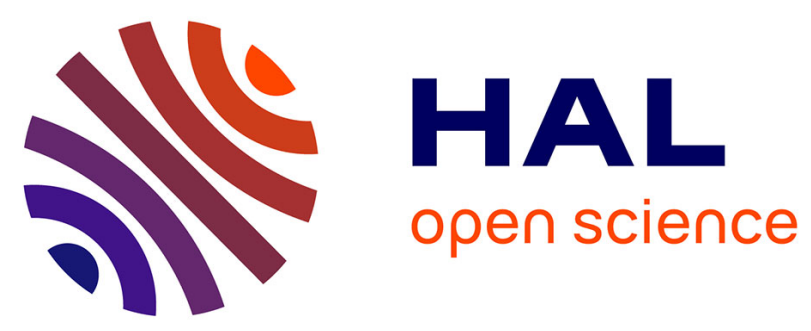

\title{
Is the Bethe-Salpeter Formalism Accurate for Excitation Energies? Comparisons with TD-DFT, CASPT2, and EOM-CCSD?
}

\author{
Denis Jacquemin, Ivan Duchemin, Xavier Blase
}

\section{- To cite this version:}

Denis Jacquemin, Ivan Duchemin, Xavier Blase. Is the Bethe-Salpeter Formalism Accurate for Excitation Energies? Comparisons with TD-DFT, CASPT2, and EOM-CCSD?. Journal of Physical Chemistry Letters, 2017, 8 (7), pp.1524. 10.1021/acs.jpclett.7b00381 . hal-01550200

\author{
HAL Id: hal-01550200 \\ https://hal.science/hal-01550200
}

Submitted on 7 Oct 2019

HAL is a multi-disciplinary open access archive for the deposit and dissemination of scientific research documents, whether they are published or not. The documents may come from teaching and research institutions in France or abroad, or from public or private research centers.
L'archive ouverte pluridisciplinaire HAL, est destinée au dépôt et à la diffusion de documents scientifiques de niveau recherche, publiés ou non, émanant des établissements d'enseignement et de recherche français ou étrangers, des laboratoires publics ou privés. 


\title{
Is Bethe-Salpeter Accurate for Excitation
}

\section{Energies? Comparisons with TD-DFT, CASPT2 and EOM-CCSD.}

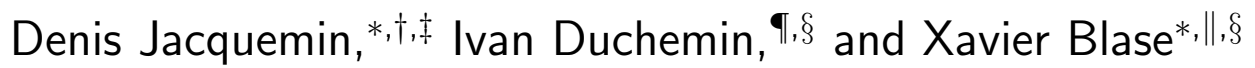 \\ $\dagger$ †aboratoire CEISAM - UMR CNR 6230, Université de Nantes, 2 Rue de la Houssinière, \\ BP 92208, 44322 Nantes Cedex 3, France \\ $\ddagger$ Institut Universitaire de France, 103 bd St. Michel, 75005 Paris Cedex 5, France \\ ๆUniv. Grenobles Alpes, CEA, INAC-MEM, L_Sim, F-38000 Grenoble, France \\ $\S$ Univ. Grenoble Alpes, Inst NEEL, F-38042 Grenoble, France \\ \|CNRS, Inst NEEL, F-38042 Grenoble, France
}

E-mail: Denis.Jacquemin@univ-nantes.fr; xavier.blase@neel.cnrs.fr 


\begin{abstract}
Developing ab initio approaches able to provide accurate excited-state energies at a reasonable computational cost is one of the biggest challenges in theoretical chemistry. In that framework the Bethe-Salpeter equation approach, combined with the $G W$ exchange-correlation self-energy, that maintains the same scaling with system size as TD-DFT, has recently been the focus of a rapidly increasing number of applications in molecular chemistry. Using a recently-proposed set encompassing excitation energies of many kinds [J. Phys. Chem. Lett., 7 (2016), 586-591], we investigate here the performances of BSE/GW. We compare these results to CASPT2, EOM-CCSD, and TD-DFT data and show that BSE/GW provides an accuracy comparable to the two wavefunction methods. It is particularly remarkable that the $\mathrm{BSE} / G W$ is equally efficient for valence, Rydberg and charge-transfer excitations. In contrast, it provides a poor description of triplet excited-states, for which EOM-CCSD and CASPT2 clearly outperform BSE $/ G W$. This contribution therefore supports the use of the BetheSalpeter approach for spin-conserving transitions.
\end{abstract}

\title{
Graphical TOC Entry
}

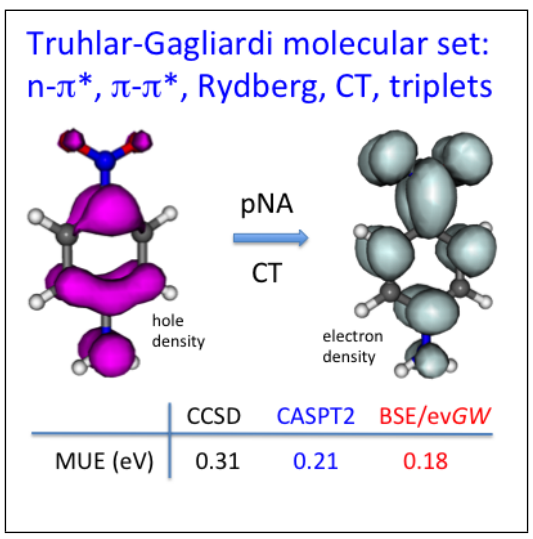


The determination of excited-state (ES) energies and properties is certainly a field in which theoretical chemistry can play a major role, because ES are both hard to capture with experimental methods and directly related to many key applications, e.g., solar energy conversion, photocatalysis, and optical information storage. Today, when one wishes to model optical spectra or other ES properties for real-life molecules, one generally turns towards TimeDependent Density Functional Theory (TD-DFT), and more precisely, to its linear-response adiabatic formulation. ${ }^{1}$ Although this method, that benefits from a formal $\mathcal{O}\left(N^{4}\right)$ scaling with system size, has been very successful, ${ }^{2,3}$ it suffers from several limitations. Amongst these drawbacks, are the impossibility to model ES with a significant multiple excitation character and the inadequacy of TD-DFT for multiconfigurational cases. In addition, TDDFT's excitation energies tend to significantly depend on the selected exchange-correlation functional. In practice, one can often find a functional that is suited for a given ES, e.g., one can select a range-separated hybrid when studying an electronic transition presenting a significant charge-transfer (CT) character, but it remains difficult to obtain a balanced description of ES of different characters (local, Rydberg, CT...) within the same compound. To achieve such description, one has to go for more refined approaches, that are unfortunately more computationally demanding. Though there have been many important developments in the field recently, ${ }^{4-6}$ multi-reference methods, such as the accurate CASPT2 or MRCI theories, remain often too expensive for tackling large conjugated molecules and, to go beyond TD-DFT, one often turns towards electron-correlated single-reference wavefunction schemes. In this vein, the most effective approach is probably $\operatorname{ADC}(2),{ }^{7}$ that delivers results similar to the ones of the more computationally involved CC2 approach, ${ }^{7-9}$ but nevertheless implies a formal $\mathcal{O}\left(N^{5}\right)$ scaling with system size. Though both $\mathrm{ADC}(2)$ and $\mathrm{CC} 2$ have the obvious advantage of not requiring the selection of an exchange-correlation functional, the obtained accuracy does not necessarily outperform its TD-DFT counterpart for excitation energies. ${ }^{10,11}$ To go further, one can use (EOM-)CCSD or $\mathrm{ADC}(3)$, but these approaches are limited by their $\mathcal{O}\left(N^{6}\right)$ scaling. Another alternative, that has been enjoying a rapidly increasing interest 
in molecular chemistry lately, ${ }^{12-18}$ is the Bethe-Salpeter equation (BSE) approach, ${ }^{19-21}$ that is a specific implementation of Green's function many-body perturbation theory (MBPT). ${ }^{21}$ BSE has also been notably used to model polyacenes. ${ }^{22-24}$ The BSE formalism relies in particular on accurate occupied/virtual energy levels, calculated within the $G W$ formalism, ${ }^{25}$ and the explicit nonlocal electron-hole interaction mediated by the screened Coulomb potential $\left(W\left(\mathbf{r}, \mathbf{r}^{\prime}\right)\right)$. This latter interaction explains in particular that charge-transfer excitations are properly described. ${ }^{26,27}$ Further, BSE has been shown to accurately reproduce excitation energies in cyanine derivatives, for which TD-DFT calculations fail independently of the use of global or range-separated hybrids, ${ }^{28}$ and several transitions with multiple-excitation character were observed to be better described than within TDDFT. ${ }^{29}$ Finally, while proceeding technically as a perturbative correction based on input DFT Kohn-Sham eigenstates, that depend on the chosen exchange-correlation functional, the BSE results become (almost) independent of the selected starting point when adopting a (partially) self-consistent $G W$ method, while conserving TD-DFT's $\mathcal{O}\left(N^{4}\right)$ scaling and hence, TD-DFT's computational efficiency. ${ }^{17}$

To date, there have been only a few "general" benchmarks devoted to BSE/ $G W$, , $, 14,29-31$ and aiming at assessing the pros and cons of this approach for molecular applications. Two strategies have been principally used. On the one hand, comparisons with vertical excitation energies determined with highly-correlated reference data obtained at the CASPT2 and CC3 levels on small molecules, ${ }^{32,33}$ have been carried out. ${ }^{29,31}$ These comparisons have highlighted that performing BSE on top of eigenstates obtained through a perturbative $G W$ correction of the DFT results, i.e., using BSE $/ G_{0} W_{0}$, implies a significant dependency on the selected DFT functional. Besides, such comparisons are inherently limited by the size of the considered compounds, e.g., charge-transfer excited-states cannot be evaluated on tiny compounds. On the other hand, our groups have performed comparisons of BSE $/ G W$ 0-0 energies with experimental references. ${ }^{9}$ While this approach allows to consider larger molecules, it is also limited, e.g., only fluorescent molecules can be treated and an approx- 
imate model accounting for environmental effects has to be used, so that decomposing the total deviations into its different contributions is not possible. In the present contribution, we provide a complementary light by using the recently proposed Truhlar-Gagliardi (TG) set of electronic transitions. ${ }^{6}$ This set is well balanced as it encompasses local, Rydberg and CT states, considers both spin-conserving and singlet-triplet transitions, and does not imply the selection of a specific environmental model. It was originally designed by these authors to assess the performances of their multiconfiguration pair-density functional theory (MC-PDFT) and they concluded that this approach was both balanced (similar deviations for all families of states) and as accurate as CASPT2 ${ }^{6}$ Herein, we use exactly the same set of compounds and show that the BSE/evGW method (in which a partially self-consistent approach is used for the $G W$ eigenvalues) is also a very competitive scheme compared to both CASPT2 and EOM-CCSD, benefitting from the facts that its computational requirements are much smaller than these two approaches, and that no active space needs to be defined (in contrast with all multiconfigurational schemes).

Our main results are listed in Table 1 and have been obtained with the aug-cc-pVTZ basis set but for the EOM-CCSD data on the two largest compounds (see computational details). As the majority reference values used in the TG set ${ }^{6}$ are originating from experiment (see Table S-1 in the SI), we have first optimized the ground-state structures at the CCSD level using large atomic basis sets, so as to improve over previously published geometries. In the SI, we compare the TD-PBE0/aug-cc-pVTZ results computed on these geometries to the TD-PBE0 values of Ref. 6 calculated with the same method with various basis sets and show that they are similar.

In a second step, we have compared the TD-DFT results obtained with two functionals, namely M06 and M06-2X, to the corresponding BSE/ev $G W$ values determined starting with the same two functionals (see Figure 1 and the SI for the BSE/evGW@M06 values). At the TD-DFT level, going from M06 to M06-2X tends to significantly upshift the transition energies, as expected when increasing the exact exchange ratio in the functional. It is only for 
Table 1: Excitation energies determined for Truhlar-Gagliardi's set of compounds. CCSD stands for EOM-CCSD, CAM for CAM-B3LYP, and BSE for BSE/ev $G W @ M 06-2 X$. The CASPT2 values are taken from Ref. 6 and the interested reader will find details regarding the impact of the size of the active space and IEPA shift in this work. The reference values are the same as in Ref. 6, and are taken from various sources (see the SI for details). All values are in $\mathrm{eV}$. $p$ NA, DMABN and B-TCNE stand for para-nitroaniline, dimethylaminobenonitrile and benzene-tetracyanoethylene complex, respectively.

\begin{tabular}{cc|cccccc}
\hline Molecule & State & CCSD & CASPT2 & TD-M06 & TD-M06-2X & BSE & Ref. \\
\hline Acetaldehyde & ${ }^{1} A^{\prime \prime}\left(n \rightarrow \pi^{\star}\right)$ & 4.40 & 4.27 & 4.26 & 4.08 & 4.26 & 4.28 \\
Acetone & ${ }^{1} A_{2}\left(n \rightarrow \pi^{\star}\right)$ & 4.58 & 4.44 & 4.43 & 4.26 & 4.28 & 4.43 \\
Formaldehyde & ${ }^{1} A_{2}\left(n \rightarrow \pi^{\star}\right)$ & 4.06 & 3.92 & 3.89 & 3.73 & 3.87 & 4.00 \\
Pyrazine & ${ }^{1} B_{3 u}\left(n \rightarrow \pi^{\star}\right)$ & 4.32 & 4.04 & 3.86 & 3.96 & 4.09 & 3.97 \\
Pyridazine & ${ }^{1} B_{1}\left(n \rightarrow \pi^{\star}\right)$ & 4.02 & 3.67 & 3.44 & 3.65 & 3.78 & 3.60 \\
Pyridine & ${ }^{1} B_{1}\left(n \rightarrow \pi^{\star}\right)$ & 5.19 & 5.06 & 4.71 & 4.87 & 5.03 & 4.74 \\
Pyrimidine & ${ }^{1} B_{1}\left(n \rightarrow \pi^{\star}\right)$ & 4.67 & 4.38 & 4.21 & 4.44 & 4.49 & 4.18 \\
$s$-Tetrazine & ${ }^{1} B_{3 u}\left(n \rightarrow \pi^{\star}\right)$ & 2.62 & 2.56 & 2.05 & 2.22 & 2.29 & 2.25 \\
Ethylene & ${ }^{1} B_{1 u}\left(\pi \rightarrow \pi^{\star}\right)$ & 8.05 & 8.16 & 7.11 & 7.53 & 7.44 & 8.02 \\
Butadiene & ${ }^{1} B_{u}\left(\pi \rightarrow \pi^{\star}\right)$ & 6.41 & 6.51 & 5.48 & 5.85 & 5.87 & 6.21 \\
Benzene & ${ }^{1} B_{2 u}\left(\pi \rightarrow \pi^{\star}\right)$ & 5.22 & 4.83 & 5.28 & 5.58 & 5.21 & 4.90 \\
& ${ }^{3} B_{1 u}\left(\pi \rightarrow \pi^{\star}\right)$ & 4.02 & 3.98 & 3.70 & 4.33 & 3.57 & 4.12 \\
Naphthalene & ${ }^{1} B_{3 u}\left(\pi \rightarrow \pi^{\star}\right)$ & 4.48 & 4.21 & 4.33 & 4.70 & 4.41 & 4.00 \\
& ${ }^{3} B_{2 u}\left(\pi \rightarrow \pi^{\star}\right)$ & 3.12 & 3.18 & 2.72 & 3.22 & 2.69 & 3.11 \\
Furan & ${ }^{1} B_{2}\left(\pi \rightarrow \pi^{\star}\right)$ & 6.57 & 6.32 & 5.78 & 6.23 & 6.01 & 6.06 \\
& ${ }^{3} B_{2}\left(\pi \rightarrow \pi^{\star}\right)$ & 4.22 & 4.08 & 3.82 & 4.30 & 3.50 & 4.17 \\
Hexatriene & ${ }^{1} B_{u}\left(\pi \rightarrow \pi^{\star}\right)$ & 5.55 & 5.32 & 4.64 & 4.95 & 4.98 & 4.93 \\
& ${ }^{3} B_{u}\left(\pi \rightarrow \pi^{\star}\right)$ & 2.78 & 2.67 & 2.24 & 2.67 & 2.22 & 2.69 \\
Water & Ryd. Singlet $2 p_{x} \rightarrow 3 s$ & 7.63 & 7.61 & 6.54 & 7.48 & 7.44 & 7.40 \\
& Ryd. Triplet $2 p_{x} \rightarrow 3 s$ & 7.24 & 7.19 & 6.29 & 7.15 & 6.80 & 7.00 \\
pNA & $\mathrm{CT}{ }^{1} A_{1}\left(\pi \rightarrow \pi^{\star}\right)$ & 4.69 & 4.62 & 4.05 & 4.51 & 4.36 & 4.30 \\
DMABN & $\mathrm{CT}{ }^{1} A_{1}\left(\pi \rightarrow \pi^{\star}\right)$ & 5.04 & 4.87 & 4.64 & 4.95 & 4.80 & 4.57 \\
B-TCNE & $\mathrm{CT}{ }^{1} A_{1}\left(\pi \rightarrow \pi^{\star}\right)$ & 3.99 & 3.84 & 2.21 & 2.96 & 3.42 & 3.59 \\
\hline
\end{tabular}

the $n \rightarrow \pi^{\star}$ transitions of acetaldehyde, acetone and formaldehyde that the opposite effect is found. On average, the increase attains $0.34 \mathrm{eV}$ and is particularly strong for the two Rydberg transitions as well as for the B-TCNE CT excited-state, a trend consistent with literature. ${ }^{34,35}$ In contrast, the impact of the functional on the TD-DFT $n \rightarrow \pi^{\star}$ transition energies is more limited, which also fits the conclusions of previous reports. ${ }^{36}$ Let us now turn towards the $\mathrm{BSE} / \mathrm{ev} G W$ results. In this case, an average upshift is also observed, but is much smaller, $0.09 \mathrm{eV}$ on average. This confirms that the partial self-consistent ev $G W$ 
procedure washes out most of the functional dependency, and this improvement is particularly impressive for the $\pi \rightarrow \pi^{\star}$, Rydberg and CT states that are much less sensitive to the starting functional than with TD-DFT. Nevertheless, small variations related to the frozen eigenvectors pertains with BSE/evGW. From Figure 1, one notices that these variations remain sizable for the $n \rightarrow \pi^{\star}$ transitions, that is they remain of the same order of magnitude as with TD-DFT. Comparing the BSE/evGW singlet-singlet and singlet-triplet transitions in a given compound, one also notices that the latter are more dependent on the selected functional - but less dependent than with TD-DFT - a conclusion that is inline with our most recent study. ${ }^{37}$ 


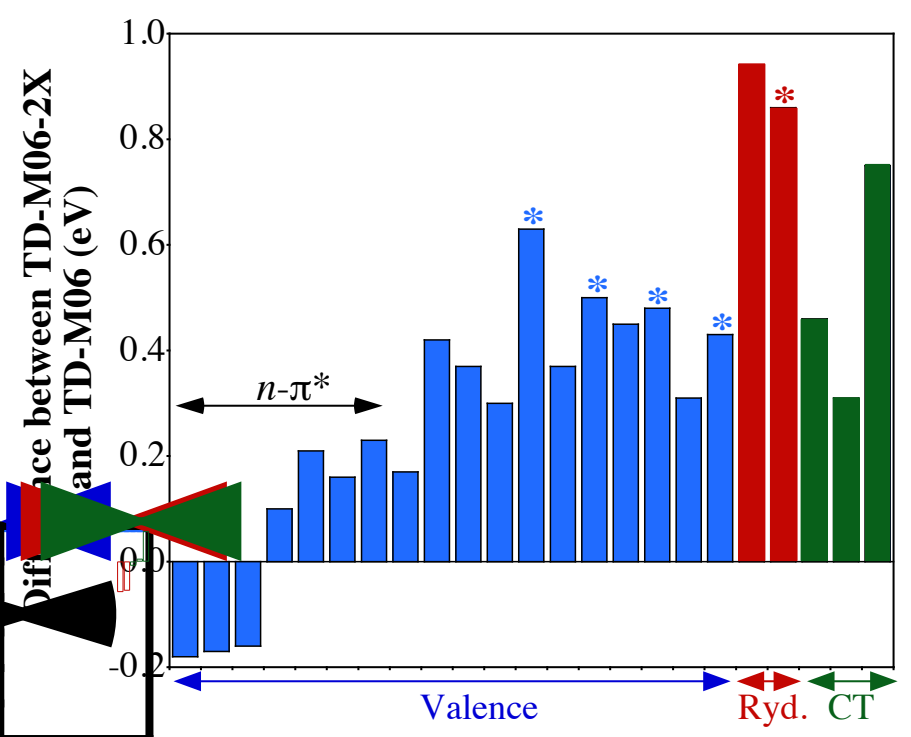

Excitation number

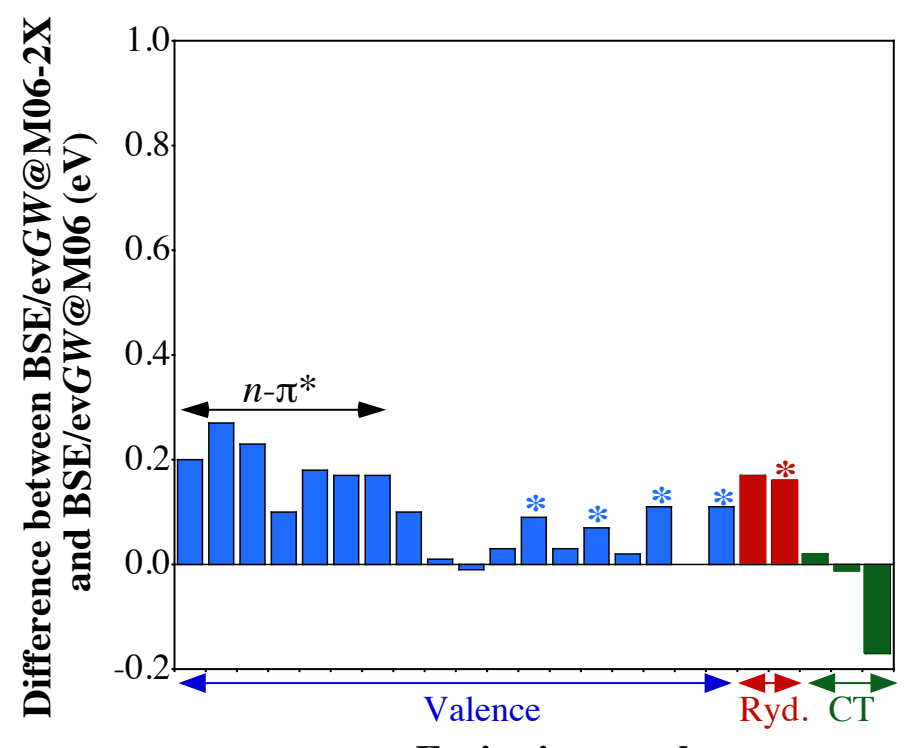

Excitation number

Figure 1: Variation of the excitation energies when changing the functional from M06 to M06-2X at the TD-DFT (top) and BSE/evGW (bottom) levels. The states are ordered as in Table 1. The blue, red and green histograms correspond to valence, Rydberg and CT transitions, respectively. The stars indicated the singlet-triplet transitions.

We now turn towards comparisons with reference values for the TG set. Table 2 lists the mean signed error (MSE), mean unsigned error (MUE) and linear determination coefficient obtained for selected methods. Histogram plots and complementary data can be found in the SI. Let us first examine the results of the two wavefunction approaches, namely EOM-CCSD 
Table 2: Mean signed and unsigned errors given in $\mathrm{eV}$ for different subsets of excitations. The CASPT2 and MC-PDFT values are from Ref. 6. At the bottom of the the Table, we provide the linear determination coefficient, $R^{2}$, obtained by comparing the full set of data to the reference values.

\begin{tabular}{cc|cccccc}
\hline Set & Error & CCSD & CASPT2 & MC-PDFT $^{a}$ & TD-M06 & TD-M06-2X & BSE/evGW@M06-2X \\
\hline Valence & MSE & 0.26 & 0.11 & -0.03 & -0.21 & 0.05 & -0.09 \\
& MUE & 0.27 & 0.15 & 0.29 & 0.29 & 0.22 & 0.28 \\
Rydberg & MSE & 0.24 & 0.20 & 0.07 & -0.78 & 0.11 & -0.08 \\
& MUE & 0.24 & 0.20 & 0.07 & 0.78 & 0.11 & 0.12 \\
CT & MSE & 0.42 & 0.29 & -0.21 & -0.52 & -0.01 & 0.04 \\
& MUE & 0.42 & 0.29 & 0.24 & 0.57 & 0.40 & 0.15 \\
\hline Total $^{b}$ & MSE & 0.30 & 0.20 & -0.06 & -0.50 & 0.05 & -0.05 \\
& MUE & 0.31 & 0.21 & 0.20 & 0.55 & 0.25 & 0.18 \\
\hline$R^{2}$ & & 0.982 & 0.989 & 0.932 & 0.919 & 0.952 & 0.953 \\
\hline
\end{tabular}

$a_{\text {tPBE functional. }}{ }^{b}$ Computed as $1 / 3$ Valence $+1 / 3$ Rydberg $+1 / 3$ CT, following Ref. 6 .

and CASPT2. Both deliver very large correlations with experiment, and positive MSE for all subsets of excitations, that is, they tend to overestimate the reference transition energies but provide very consistent trends (see also Figures S4 and S5 in the SI). In the literature, there has been contrasted reports regarding the accuracy of EOM-CCSD for valence and Rydberg states, ${ }^{10,38}$ and our results indicate that the errors can be quite significant for CT transitions, a result not highlighted before to the very best of our knowledge. As expected, CASPT2 delivers accurate data with MUE as small as $0.15 \mathrm{eV}$ for valence excitations, valuable results for Rydberg states and slightly less satisfying predictions for CT states. As can be seen in Table S-6, both EOM-CCSD and CAS-PT2 are accurate for both spin-conserving and spin-changing transitions. Of the two tested functionals, M06-2X is undoubtedly the most satisfying in the TD-DFT framework, with a small MUE for valence states, but quite large deviations for the CT states, as expected for a global hybrid. Nevertheless, we note that the range-separated CAM-B3LYP does not improve significantly the CT description in the present case (see Table S-5). The correlation with experiment provided by TD-M06-2X is smaller than its wavefunction counterparts but remains large $\left(R^{2}>0.95\right)$. Clearly TD-M06$2 \mathrm{X}$ is a cost-effective method but for $\mathrm{CT}$ transitions, a conclusion in agreement with previous 
TD-DFT benchmarks. ${ }^{39-43}$ In contrast, TD-M06 strongly undershoots both Rydberg and CT states, while TD-PBE0 yields, as expected, ${ }^{44}$ rather accurate Rydberg states (Table S-5). BSE/evGW@M06-2X improves very significantly the accuracy of CT states compared to TD-M06-2X, and stands as the most accurate method in Table 2 for this class of transitions. It also provides accurate Rydberg transition energies. For the valence states, the average deviations remain rather large, about twice the one of CAS-PT2, but similar to the EOMCCSD and MC-PDFT deviations. By analyzing more closely the BSE results (see Figure S-2 and Table S-6), we notice that these larger errors can be mainly ascribed to, on the one hand, the singlet-triplet transitions for which BSE is clearly much less accurate than most other methods, ${ }^{37}$ and, on the other hand, ethylene, a tiny molecule for which BSE is known to be inaccurate. ${ }^{29}$ Overall, using TG's formula (see footnote $b$ in Table 2) to estimate the total deviation, the Bethe-Salpeter approach appears to be the method delivering both the smallest MSE and the smallest MUE. Whilst such conclusions should be ascertained in the future for a larger set of molecules, it is certainly remarkable that no subset of transitions yield BSE MUE exceeding $0.3 \mathrm{eV}$, a success that is shared by CASPT2 and MC-PDFT only, two multi-configurational approaches.

To provide additional insights into the success of the Bethe-Salpeter approach, we have performed aug-cc-pVTZ CCSD(T) calculations of the HOMO-LUMO gap, excluding the largest DMABN and B-CTCNE systems (Table S-4 in the SI), showing that the gaps predicted by the evGW@M06 and evGW@M06-2X methods are within $0.13 \mathrm{eV}$ and $0.21 \mathrm{eV}$ (MSE) of the $\operatorname{CCSD}(\mathrm{T})$ reference, respectively. This $0.08 \mathrm{eV}$ MSE difference between HOMOLUMO gaps can be compared with the $0.10 \mathrm{eV}$ MSE difference between the corresponding BSE/evGW@M06 and BSE/evGW@M06-2X calculations for singlet excitations, confirming the good average correlation between the $G W$ gap and the corresponding BSE excitation energies. ${ }^{31}$ Consistently with Ref. 31, we note however that the $G W$ and BSE/GW MSEs are not identical, with a small difference of $0.2-0.3 \mathrm{eV}$, so that it cannot be strictly concluded that an exact BSE/ $G W$ excitation energy corresponds to an exact $G W$ HOMO-LUMO gap. 
Further, beyond average correlations, rather large variations exist, such as in the case of ethylene for which the error on the BSE excitation energy $(0.58 \mathrm{eV})$ is much larger than that on the $G W$ gap $(0.09 \mathrm{eV})$, calling for further exploration of the pros and cons of the BSE formalism applied to organic systems.

In short, using the representative TG set of transition energies, we have shown that BSE/evGW is an effective method for studying the excitation energies of molecules. In spite of its singlereference nature, its small dependency on the selected functional and its favorable $\mathcal{O}\left(N^{4}\right)$ scaling, this theoretical method provides a balanced description of all families of excitedstates, a success only achieved previously with multi-reference approaches and/or computationally expensive high-order wavefunction schemes. The obtained average unsigned deviation is comparable to the one of CASPT2, but the correlation coefficient with the reference value is smaller than with CASPT2 or EOM-CCSD. This effect is mainly due to the presence of singlet-triplet transitions in the TG sets, a subset for which BSE/ev $G W$ results are poor. We can therefore advocate the use of $\mathrm{BSE} / \mathrm{ev} G W$ for investigating spin-conserving transitions in large molecular systems. Of course, BSE/evGW has also its limitations, e.g., is it not suited for significantly multi-configurational cases as CASPT2, and, it is not systematically improvable by increasing the perturbation order as CC.

As a further consideration, we emphasise that the present BSE/ev $G W$ formalism proceeds as a perturbation theory starting from the mono-determinental Kohn-Sham ansatz groundstate wavefunction. Further, we performed BSE calculation exploiting the statically screened Coulomb potential, namely we adopted the analog of the adiabatic approximation used in TD-DFT. As such, the consideration that BSE calculations with dynamical screening could help in describing transitions with multiple excitation character ${ }^{45}$ cannot be invoked here. It is therefore difficult to conclude at the present stage that the improvement over TD-DFT calculations for the present set of molecules stems potentially from a better description of cases presenting a multi-determinental character. We can rather conclude that the use of the calculated microscopic screened-Coulomb potential $W\left(r, r^{\prime}\right)$ offers a much more flexible 
description of the short, medium and, long range electron-hole interactions, as compared to global or range-separated hybrid functionals that rely on a more "rigid" functional form. While the present study advocates the Bethe-Salpeter formalism for its remarkable accuracy and favourable $\mathcal{O}\left(N^{4}\right)$ scaling, one additional conclusion is that the standard adiabatic TDDFT approach may still be improved by pursuing the search for better hybrid functionals.

\section{Computational Details}

But for the B-TCNE structure that was taken in the literature, ${ }^{46}$ the ground-state geometries of all compounds was optimized at the CCSD level with a large atomic basis set, typically def2-TZVPP (see the SI for Cartesian coordinates). The optimizations were performed with the Gaussian09 program, ${ }^{47}$ imposing a tight convergence threshold on the forces and using $10^{-8}$ and $10^{-10}$ a.u. convergence thresholds on the CCSD and SCF energies, respectively. The EOM-CCSD and TD-DFT calculations were performed, with the same program, applying Dunning's aug-cc-pVTZ atomic basis set but for the coupled-cluster calculations on DMABN and B-TCNE for which the aug-cc-pVDZ atomic basis set was used for obvious computational reasons. The $\operatorname{CCSD}(\mathrm{T})$ calculations in the SI were performed with Gaussian09 as well. ${ }^{47}$ The $G W$ and BSE calculations use aug-cc-pVTZ and have been achieved with the FIESTA package ${ }^{48}$ applying the Coulomb-fitting resolution-of-identity (RI-V) technique. It is important to stress that we used here the so-called evGW level, that is, we self-consistently converged the occupied/virtual $G W$ energy levels while keeping the input DFT eigenfunctions frozen. We corrected at the $G W$ level, all valence occupied DFT levels and twice that amount of virtual levels. The BSE calculations were made with the "full" matrix, i.e., we do not apply the Tamm-Dancoff approximation here. For the $G W$ calculations, the starting DFT eigenstates were generated with NWChem ${ }^{49}$ using the M06 and M06-2X hybrid functionals, ${ }^{50}$ and applying the $x$ fine integration grid, as well as $10^{-7}$ a.u. and $10^{-6}$ a.u. convergence thresholds for the total energies and densities, respectively. 


\section{Acknowledgement}

D.J. acknowledges the European Research Council (ERC) and the Région des Pays de la Loire for financial support in the framework of a Starting Grant (Marches - 278845) and the LumoMat project, respectively. X.B. and I.D. acknowledge funding from the European Union Horizon 2020 research and innovation programme under Grant Agreement No. 646176 (EXTMOS). This research used resources of i) the GENCI-CINES/IDRIS; ii) CCIPL (Centre de Calcul Intensif des Pays de Loire); iii) a local Troy cluster and iv) HPC resources from ArronaxPlus (grant ANR-11-EQPX-0004 funded by the French National Agency for Research).

\section{Supporting Information Available}

Geometries used in all calculations. Oirgin of the reference values used here. Comparisons between the TD-PBE0 transition energies computed on our CCSD geometries and taken from Ref. 6. BSE/evGW@M06 and TD-CAM-B3LYP transition energies. ev $G W$ gaps. Error plots for selected methods. Additional error analysis.

\section{References}

(1) Ullrich, C. Time-Dependent Density-Functional Theory: Concepts and Applications; Oxford Graduate Texts; Oxford University Press: New York, 2012.

(2) Laurent, A. D.; Adamo, C.; Jacquemin, D. Dye Chemistry with Time-Dependent Density Functional Theory. Phys. Chem. Chem. Phys. 2014, 16, 14334-14356.

(3) Santoro, F.; Jacquemin, D. Going Beyond the Vertical Approximation with TimeDependent Density Functional Theory. WIREs Comput. Mol. Sci. 2016, 6, 460-486.

(4) Ren, J.; Yi, Y.; Shuai, Z. Inner Space Perturbation Theory in Matrix Product States: 
Replacing Expensive Iterative Diagonalization. J. Chem. Theory Comput. 2016, 12, $4871-4878$.

(5) Knecht, S.; Keller, S.; Autschbach, J.; Reiher, M. A Nonorthogonal State-Interaction Approach for Matrix Product State Wave Functions. J. Chem. Theory Comput. 2016, 12, 5881-5894.

(6) Hoyer, C. E.; Ghosh, S.; Truhlar, D. G.; Gagliardi, L. Multiconfiguration Pair-Density Functional Theory Is as Accurate as CASPT2 for Electronic Excitation. J. Phys. Chem. Lett. 2016, 7, 586-591.

(7) Dreuw, A.; Wormit, M. The Algebraic Diagrammatic Construction Scheme for the Polarization Propagator for the Calculation of Excited States. WIREs Comput. Mol. Sci. 2015, 5, 82-95.

(8) Winter, N. O. C.; Graf, N. K.; Leutwyler, S.; Hattig, C. Benchmarks for 0-0 Transitions of Aromatic Organic Molecules: DFT/B3LYP, ADC(2), CC2, SOS-CC2 and SCS-CC2 Compared to High-Resolution Gas-Phase Data. Phys. Chem. Chem. Phys. 2013, 15, 6623-6630.

(9) Jacquemin, D.; Duchemin, I.; Blase, X. 0-0 Energies Using Hybrid Schemes: Benchmarks of TD-DFT, CIS(D), ADC(2), CC2 and BSE/GW formalisms for 80 Real-Life Compounds. J. Chem. Theory Comput. 2015, 11, 5340-5359.

(10) Schreiber, M.; Silva-Junior, M. R.; Sauer, S. P. A.; Thiel, W. Benchmarks for Electronically Excited States: CASPT2, CC2, CCSD and CC3. J. Chem. Phys. 2008, 128, 134110.

(11) Silva-Junior, M. R.; Schreiber, M.; Sauer, S. P. A.; Thiel, W. Benchmarks for Electronically Excited States: Time-Dependent Density Functional Theory and Density Functional Theory Based Multireference Configuration Interaction. J. Chem. Phys. 2008, 129, 104103. 
(12) Blase, X.; Boulanger, P.; Bruneval, F.; Fernandez-Serra, M.; Duchemin, I. GW and Bethe-Salpeter Study of Small Water Clusters. J. Chem. Phys. 2016, 144.

(13) Rebolini, E.; Toulouse, J. Range-Separated Time-Dependent Density-Functional Theory With a Frequency-Dependent Second-Order Bethe-Salpeter Correlation Kernel. J. Chem. Phys. 2016, 144, 094107.

(14) Jacquemin, D.; Duchemin, I.; Blondel, A.; Blase, X. Assessment of the Accuracy of the Bethe-Salpeter (BSE/GW) Oscillator Strengths. J. Chem. Theory Comput. 2016, 12, 3969-3981.

(15) Ziaei, V.; Bredow, T. GW-BSE Approach on S1 Vertical Transition Energy of Large Charge Transfer Compounds: A Performance Assessment. J. Chem. Phys. 2016, 145, 174305.

(16) Hung, L.; da Jornada, F. H.; Souto-Casares, J.; Chelikowsky, J. R.; Louie, S. G.; Ögüt, S. Excitation Spectra of Aromatic Molecules Within a Real-Space $G W$-BSE Formalism: Role of Self-Consistency and Vertex Corrections. Phys. Rev. B 2016, 94 , 085125 .

(17) Krause, K.; Klopper, W. Implementation of the BetheSalpeter Equation in the TURBOMOLE Program. J. Comput. Chem. 2017, 38, 383-388.

(18) Azarias, C.; Duchemin, I.; Blase, X.; Jacquemin, D. Bethe-Salpeter Study of Cationic Dyes: Comparisons with ADC(2) and TD-DFT. J. Chem. Phys. 2017, 146, 034301.

(19) Sham, L. J.; Rice, T. M. Many-Particle Derivation of the Effective-Mass Equation for the Wannier Exciton. Phys. Rev. 1966, 144, 708-714.

(20) Strinati, G. Dynamical Shift and Broadening of Core Excitons in Semiconductors. Phys. Rev. Lett. 1982, 49, 1519-1522. 
(21) Onida, G.; Reining, L.; Rubio, A. Electronic Excitations: Density-Functional Versus Many-Body Green's-Function Approaches. Rev. Mod. Phys. 2002, 74, 601-659.

(22) Tiago, M. L.; Chelikowsky, J. R. Optical excitations in organic molecules, clusters, and defects studied by first-principles Green's function methods. Phys. Rev. B 2006, 73, 205334 .

(23) Coto, P. B.; Sharifzadeh, S.; Neaton, J. B.; Thoss, M. Low-Lying Electronic Excited States of Pentacene Oligomers: A Comparative Electronic Structure Study in the Context of Singlet Fission. J. Chem. Theory Comput. 2015, 11, 147-156, PMID: 26574213.

(24) Leng, X.; Feng, J.; Chen, T.; Liu, C.; Ma, Y. Optical Properties of Acene Molecules and Pentacene Crystal From the Many-Body Green'S Function Method. Phys. Chem. Chem. Phys. 2016, 18, 30777-30784.

(25) Hedin, L. New Method for Calculating the One-Particle Green's Function with Application to the Electron-Gas Problem. Phys. Rev. A 1965, 139, 796-823.

(26) Duchemin, I.; Deutsch, T.; Blase, X. Short-Range to Long-Range Charge-Transfer Excitations in the Zincbacteriochlorin-Bacteriochlorin Complex: A Bethe-Salpeter Study. Phys. Rev. Lett. 2012, 109, 167801.

(27) Duchemin, I.; Blase, X. Resonant Hot Charge-Transfer Excitations in FullerenePorphyrin Complexes: Many-Body Bethe-Salpeter Study. Phys. Rev. B 2013, 87, 245412.

(28) Boulanger, P.; Jacquemin, D.; Duchemin, I.; Blase, X. Fast and Accurate Electronic Excitations in Cyanines with the Many-Body Bethe-Salpeter Approach. J. Chem. Theory Comput. 2014, 10, 1212-1218.

(29) Jacquemin, D.; Duchemin, I.; Blase, X. Benchmarking the Bethe-Salpeter Formalism on a Standard Organic Molecular Set. J. Chem. Theory Comput. 2015, 11, 3290-3304. 
(30) Körbel, S.; Boulanger, P.; Duchemin, I.; Blase, X.; Marques, M. A. L.; Botti, S. Benchmark Many-Body GW and Bethe-Salpeter Calculations for Small Transition Metal Molecules. J. Chem. Theory Comput. 2014, 10, 3934-3943.

(31) Bruneval, F.; Hamed, S. M.; Neaton, J. B. A Systematic Benchmark of the Ab Initio Bethe-Salpeter Equation Approach for Low-Lying Optical Excitations of Small Organic Molecules. J. Chem. Phys. 2015, 142, 244101.

(32) Silva-Junior, M. R.; Sauer, S. P. A.; Schreiber, M.; Thiel, W. Basis Set Effects on Coupled Cluster Benchmarks of Electronically Excited States: CC3, CCSDR(3) and CC2. Mol. Phys. 2010, 108, 453-465.

(33) Silva-Junior, M. R.; Schreiber, M.; Sauer, S. P. A.; Thiel, W. Benchmarks of Electronically Excited States: Basis Set Effecs Benchmarks of Electronically Excited States: Basis Set Effects on CASPT2 Results. J. Chem. Phys. 2010, 133, 174318.

(34) Dreuw, A.; Head-Gordon, M. Failure of Time-Dependent Density Functional Theory for Long-Range Charge-Transfer Excited States: the Zincbacteriochlorin-Bacteriochlorin and Bacteriochlorophyll-Spheroidene Complexes. J. Am. Chem. Soc. 2004, 126, 40074016.

(35) Peach, M. J. G.; Benfield, P.; Helgaker, T.; Tozer, D. J. Excitation Energies in Density Functional Theory: an Evaluation and a Diagnostic Test. J. Chem. Phys. 2008, 128, 044118.

(36) Jacquemin, D.; Perpète, E. A.; Vydrov, O. A.; Scuseria, G. E.; Adamo, C. Assessment of long-range corrected functionals performance for $n \rightarrow \pi^{\star}$ transitions in organic dyes. J. Chem. Phys. 2007, 12\%, 094102.

(37) Jacquemin, D.; Duchemin, I.; Blondel, A.; Blase, X. Benchmark of Bethe-Salpeter for Triplet Excited-States. J. Chem. Theory Comput. 2017, 13, 767-783. 
(38) Caricato, M.; Trucks, G. W.; Frisch, M. J.; Wiberg, K. B. Electronic Transition Energies: A Study of the Performance of a Large Range of Single Reference Density Functional and Wave Function Methods on Valence and Rydberg States Compared to Experiment. J. Chem. Theory Comput. 2010, 6, 370-383.

(39) Jacquemin, D.; Perpète, E. A.; Ciofini, I.; Adamo, C. Assessment of Functionals for TD-DFT Calculations of Singlet-Triplet Transitions. J. Chem. Theory Comput. 2010, 6, 1532-1537.

(40) Jacquemin, D.; Perpète, E. A.; Ciofini, I.; Adamo, C.; Valero, R.; Zhao, Y.; Truhlar, D. G. On the Performances of the M06 Family of Density Functionals for Electronic Excitation Energies. J. Chem. Theory Comput. 2010, 6, 2071-2085.

(41) Isegawa, M.; Peverati, R.; Truhlar, D. G. Performance of Recent and High-Performance Approximate Density Functionals for Time-Dependent Density Functional Theory Calculations of Valence and Rydberg Electronic Transition Energies. J. Chem. Phys. 2012, $137,244104$.

(42) Jacquemin, D.; Planchat, A.; Adamo, C.; Mennucci, B. A TD-DFT Assessment of Functionals for Optical 0-0 Transitions in Solvated Dyes. J. Chem. Theory Comput. 2012, 8, 2359-2372.

(43) Leang, S. S.; Zahariev, F.; Gordon, M. S. Benchmarking the Performance of TimeDependent Density Functional Methods. J. Chem. Phys. 2012, 136, 104101.

(44) Ciofini, I.; Adamo, C. Accurate evaluation of valence and low-lying rydberg states with standard time-dependent density functional theory. J. Phys. Chem. A 2007, 111, $5549-5556$.

(45) Sangalli, D.; Romaniello, P.; Onida, G.; Marini, A. Double excitations in correlated systems: A many-body approach. J. Chem. Phys. 2011, 134, 034115. 
(46) Stein, T.; Kronik, L.; Baer, R. Reliable Prediction of Charge Transfer Excitations in Molecular Complexes Using Time-Dependent Density Functional Theory. J. Am. Chem. Soc. 2009, 131, 2818-2820.

(47) Frisch, M. J.; Trucks, G. W.; Schlegel, H. B.; Scuseria, G. E.; Robb, M. A.; Cheeseman, J. R.; Scalmani, G.; Barone, V.; Mennucci, B.; Petersson, G. A. et al. Gaussian 09 Revision D.01. 2009; Gaussian Inc. Wallingford CT.

(48) Blase, X.; Attaccalite, C.; Olevano, V. First-Principles $G W$ Calculations for Fullerenes, Porphyrins, Phtalocyanine, and Other Molecules of Interest for Organic Photovoltaic Applications. Phys. Rev. B 2011, 83, 115103.

(49) Valiev, M.; Bylaska, E. J.; Govind, N.; Kowalski, K.; Straatsma, T. P.; Van Dam, H. J. J.; Wang, D.; Nieplocha, J.; Apra, E.; Windus, T. L. et al. NWChem: a Comprehensive and Scalable Open-Source Solution for Large Scale Molecular Simulations. Comput. Phys. Commun. 2010, 181, 1477-1489.

(50) Zhao, Y.; Truhlar, D. G. The M06 Suite of Density Functionals for Main Group Thermochemistry, Thermochemical Kinetics, Noncovalent Interactions, Excited States, and Transition Elements: Two New Functionals and Systematic Testing of Four M06-Class Functionals and 12 Other Functionals. Theor. Chem. Acc. 2008, 120, 215-241. 